\title{
Statistical Emission Image Reconstruction for Randoms-Precorrected PET Scans Using Negative Sinogram Values
}

\author{
Sangtae Ahn and Jeffrey A. Fessler
}

\begin{abstract}
Many conventional PET emission scans are corrected for accidental coincidence (AC) events, or randoms, by real-time subtraction of delayed-window coincidences, leaving only the randoms-precorrected data available for image reconstruction. The real-time precorrection compensates in mean for AC events but destroys Poisson statistics. Since the exact log-likelihood for randoms-precorrected data is inconvenient to maximize, practical approximations are desirable for statistical image reconstruction. Conventional approximations involve setting negative sinogram values to zero, which can induce positive systematic biases, particularly for scans with low counts per ray. We propose new likelihood approximations that allow negative sinogram values without requiring zero-thresholding. We also develop monotonic algorithms for the new models by using "optimization transfer" principles. Simulation results show that our new model, $\mathrm{SP}^{-}$, is free of systematic bias yet keeps low variance. Despite its simpler implementation, the new model performs comparably to the saddle-point (SD) model which has previously shown the best performance (as to systematic bias and variance) in randoms-precorrected PET emission reconstruction.
\end{abstract}

\section{INTRODUCTION}

Accidental coincidence (AC) events, also known as randoms, are a primary source of background noise in positron emission tomography (PET) [1]. PET systems usually detect coincidence events during "prompt" windows and "delayed" windows [2], and in many conventional PET scans, the prompt data are precorrected for the effects of AC events by real-time subtraction of delayed coincidences [1]. The subtraction compensates for the $\mathrm{AC}$ events in terms of the mean, but increases the variance of the data and destroys Poisson statistics [3], [4].

It would be preferable to record both prompt and randoms sinograms so that one could estimate the mean of AC events from the randoms sinogram [4]-[7] and incorporate the estimates into an appropriate model for the prompt measurement [4], [6], [8]. However, because of data storage limitations and historical momentum, many conventional PET centers store the randoms-precorrected data only [2]. We focus on the problem of reconstructing statistically emission images based

This work was supported in part by the NSF under Grant BES-9982349, by the NIH under Grant CA-60711, and by the DOE under Grant DE-FG0287ER60561.

The authors are with the Electrical Engineering and Computer Science Department, University of Michigan, Ann Arbor, MI 48109-2122 USA (email: sangtaea@umich.edu, fessler@umich.edu). on only randoms-precorrected data without access to separate prompt and randoms sinograms.

We have previously developed shifted Poisson (SP) and saddle-point (SD) models to approximate the log-likelihood of randoms-precorrected data [2], [3], [9]. We have also applied those models to emission image reconstruction [10]; the SD and SP models led to lower variance than the conventional ordinary Poisson (OP) model and the nonstatistical filtered backprojection (FBP) for high photon counts per ray whereas all the methods other than SD were of positive systematic bias for low counts per ray.

The fact that the positive systematic bias is caused by zeroing negative sinogram values [10] motivates us to propose new models, $\mathrm{SP}^{-}$and $\mathrm{OP}^{-}$, by allowing negative values. Although allowing negative values can lead to nonconcave optimization, using optimization transfer principles, we develop for the new models monotonic reconstruction algorithms ascending to local maximizers of the objective function. Simulation results show that the new $\mathrm{SP}^{-}$model is comparable, in spite of its simpler implementation, to SD which has previously shown the best performance as to systematic bias and variance.

\section{PROBLEM}

Let $\boldsymbol{Y}=\left[Y_{1} \ldots Y_{N}\right]^{\prime}$ denote the precorrected measurements for PET emission scans, where' denotes vector transpose. The precorrected measurement for the $i$ th bin is

$$
Y_{i}=Y_{i}^{\text {prompt }}-Y_{i}^{\text {delay }}
$$

where $Y_{i}^{\text {prompt }}$ and $Y_{i}^{\text {delay }}$ are the number of coincidences detected within the prompt and delayed windows, respectively. The prompts and delays can be modeled reasonably as independent Poisson random variables as follows:

$$
\begin{aligned}
Y_{i}^{\text {prompt }} & \sim \operatorname{Poisson}\left\{\sum_{j=1}^{p} a_{i j} \lambda_{j}^{\text {true }}+r_{i}+s_{i}\right\} \\
Y_{i}^{\text {delay }} & \sim \operatorname{Poisson}\left\{r_{i}\right\}
\end{aligned}
$$

where $a_{i j} \geq 0$ is the entry in the system matrix $\boldsymbol{A} ; \lambda_{j}^{\text {true }} \geq 0$ is the activity at the $j$ th voxel; and $r_{i}>0$ and $s_{i} \geq 0$ are the means of AC events and scatters, respectively. We assume that $\boldsymbol{r}=\left[r_{1} \ldots r_{N}\right]^{\prime}$ and $\boldsymbol{s}=\left[s_{1} \ldots s_{N}\right]^{\prime}$ are known.

Let $\boldsymbol{y}=\left[y_{1} \ldots y_{N}\right]^{\prime}$ be an observed realization of $\boldsymbol{Y}$. Since the measurements are independent, one can obtain the exact 
log-likelihood, ignoring constants independent of $\boldsymbol{\lambda}$, as in [3]:

$$
L(\boldsymbol{\lambda} ; \boldsymbol{Y})=\sum_{i=1}^{N} h_{i}^{\mathrm{EX}}\left(l_{i}(\boldsymbol{\lambda})\right)
$$

with

$$
l_{i}(\boldsymbol{\lambda})=\sum_{j=1}^{p} a_{i j} \lambda_{j}
$$

and

$h_{i}^{\mathrm{EX}}(l)=\log \left(\sum_{m=\left[-y_{i}\right]_{+}}^{\infty} \frac{\left(l+r_{i}+s_{i}\right)^{y_{i}+m}}{\left(y_{i}+m\right) !} \frac{r_{i}^{m}}{m !}\right)-\left(l+2 r_{i}+s_{i}\right)$

where $[x]_{+}=\max \{x, 0\}$.

For penalized-likelihood (PL) reconstruction, one must find a maximizer of the objective function

$$
\Phi(\boldsymbol{\lambda} ; \boldsymbol{Y})=L(\boldsymbol{\lambda} ; \boldsymbol{Y})-R(\boldsymbol{\lambda})
$$

over a nonnegativity constraint on the image $\lambda$, where $R$ is a regularization term. The exact log-likelihood function (4) is inconvenient to maximize, and the next section describes practical approximations to the exact log-likelihood.

\section{Approximations to EXACT Log-LiKelihood}

\section{A. Ordinary Poisson (OP) Approximation}

A simple approach that does not need an estimate of AC events $\boldsymbol{r}$ is to approximate the measurements as Poisson random variables as follows:

$$
Y_{i} \stackrel{\substack{\text { Op } \\ \text { approx. }}}{\sim} \text { Poisson }\left\{\sum_{j=1}^{p} a_{i j} \lambda_{j}^{\text {true }}+s_{i}\right\} .
$$

This model matches the first moment of $Y_{i}$ only. The loglikelihood $L^{\mathrm{OP}^{-}}$corresponding to this " $\mathrm{OP}^{-}$" approximation is of the form (4) with

$$
h_{i}^{\mathrm{OP}^{-}}(l)=y_{i} \log \left(l+s_{i}\right)-\left(l+s_{i}\right) .
$$

We assume $s_{i}>0$ in (9); otherwise, negative values $y_{i}$ would cause reconstruction algorithms to diverge since $h_{i}^{\mathrm{OP}^{-}}(0)=$ $+\infty$ for $y_{i}<0$ and $s_{i}=0$. To avoid such divergence, past studies of the OP approach [10] for emission scans have used zero-thresholded values as follows:

$$
h_{i}^{\mathrm{OP}^{+}}(l)=\left[y_{i}\right]_{+} \log \left(l+s_{i}\right)-\left(l+s_{i}\right),
$$

called the "OP" ${ }^{+}$" approximation in this paper. (Note the slightly different use of terms from [10].) The zerothresholding is natural in view of the nonnegative nature of Poisson random variables in (8). However, zero-thresholding destroys the first moment matching in (8), and the increase in the mean value of the precorrected data causes the estimators to have a positive systematic bias.

\section{B. Shifted Poisson (SP) Approximation}

An improved approximation is to match both the first and second moments as follows:

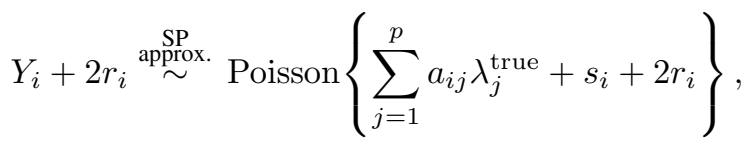

where in practice one must use an estimate $\hat{r}_{i}$. This " $\mathrm{SP}^{-}$" approximation leads to a $\log$-likelihood function $L^{\mathrm{SP}^{-}}$of the form (4) with

$$
h_{i}^{\mathrm{SP}^{-}}(l)=\left(y_{i}+2 r_{i}\right) \log \left(l+s_{i}+2 r_{i}\right)-\left(l+s_{i}+2 r_{i}\right) .
$$

Similarly, its conventional zero-thresholded version $L^{\mathrm{SP}^{+}}$ uses [10]

$$
h_{i}^{\mathrm{SP}^{+}}(l)=\left[y_{i}+2 r_{i}\right]_{+} \log \left(l+s_{i}+2 r_{i}\right)-\left(l+s_{i}+2 r_{i}\right) .
$$

The zero-thresholding again causes positive systematic bias, albeit generally less than that of $\mathrm{OP}^{+}$.

\section{Saddle-Point (SD) Approximation}

Another approach is to make a second order Taylor series approximation in the $z$-transform domain to the probability generating function and then carry out the inverse transform [11], [12]. The log-likelihood $L^{\mathrm{SD}}$ corresponding to this SD approximation [10] is of the form (4) with

$$
h_{i}^{\mathrm{SD}}(l)=y_{i} \log \left(\frac{l+s_{i}+r_{i}}{z_{i}+u_{i}(l)}\right)-l+u_{i}(l)-\frac{1}{2} \log u_{i}(l)
$$

where

$$
z_{i}= \begin{cases}y_{i}+1, & \text { for } y_{i} \geq 0 \\ y_{i}-1, & \text { for } y_{i}<0\end{cases}
$$

and

$$
u_{i}(l)=\sqrt{z_{i}^{2}+4\left(l+r_{i}+s_{i}\right) r_{i}} .
$$

The SD model for emission image reconstruction is free of systematic bias and leads to lower variance than $\mathrm{OP}^{+}[10]$.

\section{Log-likelihood for Prompt Data}

If one has access to the prompt data $\boldsymbol{Y}^{\text {prompt }}$, then one can use the log-likelihood for the prompt data in the form (4) with

$$
h_{i}^{\mathrm{PR}}(l)=y_{i}^{\mathrm{prompt}} \log \left(l+s_{i}+r_{i}\right)-\left(l+s_{i}+r_{i}\right) .
$$

We include this PR model for comparing the bias and variance of the methods for randoms-precorrected data in Section V. The PR model serves as a baseline for comparing algorithms. 


\section{Reconstruction Algorithms}

One needs an algorithm to maximize the corresponding objective function for maximum likelihood or penalizedlikelihood estimation. It is straightforward to use globally convergent (and monotonic) algorithms such as separable paraboloidal surrogates (SPS) [13] and ML-EM [14] for PR, $\mathrm{OP}^{+}, \mathrm{SP}^{+}$and $\mathrm{SD}$, all of which have concave log-likelihoods (See [15, Ch. 5] for algorithms for SD). However, the new $\mathrm{OP}^{-}$and $\mathrm{SP}^{-}$models can have nonconcave log-likelihood functions when negative sinogram values are present. The algorithms need some modifications to ensure monotonicity for the nonconcave case as well.

A large class of monotonic iterative algorithms (including SPS and ML-EM) are based on "optimization transfer" principles: at each iteration we choose a surrogate function that is easier to maximize than the original objective function, and then maximize that surrogate. To ensure monotonicity, the surrogate function is chosen so that increasing the surrogate guarantees the increase of the original objective function.

The idea for extending the algorithms to allow negative values is to choose a linear surrogate when a marginal loglikelihood is convex. That is, for $\mathrm{OP}^{-}$, if $y_{i}<0$, a tangent line to $h_{i}^{\mathrm{OP}^{-}}$at a current iterate $l_{i}^{n}$ in projection domain,

$$
q_{i}^{\mathrm{OP}^{-}}\left(l ; l_{i}^{n}\right)=\dot{h}_{i}^{\mathrm{OP}^{-}}\left(l_{i}^{n}\right)\left(l-l_{i}^{n}\right)+h_{i}^{\mathrm{OP}^{-}}\left(l_{i}^{n}\right),
$$

is a proper surrogate for $h_{i}^{\mathrm{OP}^{-}}$in light of [16, (7)] since $q_{i}^{\mathrm{OP}^{-}}$ lies below for all $l \geq 0$ due to convexity of $h_{i}^{\mathrm{OP}^{-}}$. In other words, if $q_{i}^{\mathrm{OP}^{-}}\left(l ; l_{i}^{n}\right) \geq q_{i}^{\mathrm{OP}^{-}}\left(l_{i}^{n} ; l_{i}^{n}\right)$, then $h_{i}^{\mathrm{OP}^{-}}\left(l ; l_{i}^{n}\right) \geq$ $h_{i}^{\mathrm{OP}^{-}}\left(l_{i}^{n} ; l_{i}^{n}\right)$. The same principle applies to $\mathrm{SP}^{-}$when $y_{i}+$ $2 r_{i}<0$.

We derive modified SPS applicable to $\mathrm{OP}^{-}$and $\mathrm{SP}^{-}$, using a linear surrogate (14) when needed (See [17] for derivation of modified ML-EM). We consider the PL objective function $\Phi$ in (7) with a quadratic penalty for simplicity:

$$
R(\boldsymbol{\lambda})=\frac{\beta}{2} \sum_{j=1}^{p} \sum_{k \in \mathcal{N}_{j}} \omega_{j k} \frac{\left(\lambda_{j}-\lambda_{k}\right)^{2}}{2}
$$

where $\beta \geq 0$ is a regularization parameter that controls the smoothness of the reconstructed image, $\mathcal{N}_{j}$ denotes the neighborhood of the $j$ th pixel, and $\omega_{j k}$ is a weighting factor. A monotonic SPS for $\mathrm{OP}^{-}$and $\mathrm{SP}^{-}$is readily derived following [13] with (14). The resulting algorithm differs only slightly from the ordinary SPS algorithm in [13], and uses the following iteration:

$$
\lambda_{j}^{n+1}=\left[\lambda_{j}^{n}+\frac{1}{d_{j}^{\mathrm{SPS}}\left(\boldsymbol{\lambda}^{n}\right)} \frac{\partial \Phi\left(\boldsymbol{\lambda}^{n}\right)}{\partial \lambda_{j}}\right]_{+}
$$

with

$$
d_{j}^{\mathrm{SPS}}(\boldsymbol{\lambda})=\sum_{i=1}^{N} a_{i j} a_{i} c_{i}\left(l_{i}(\boldsymbol{\lambda})\right)+2 \beta \sum_{k \in \mathcal{N}_{j}} \omega_{j k}
$$

where $a_{i}=\sum_{j=1}^{p} a_{i j}$ and

$$
c_{i}(l)= \begin{cases}2\left[h_{i}(l)-h_{i}(0)-l \dot{h}_{i}(l)\right] / l^{2}, & l>0, x_{i}>0 \\ -\ddot{h}_{i}(0), & l=0, x_{i}>0 \\ 0, & x_{i} \leq 0\end{cases}
$$

in which $h_{i}$ represents $h_{i}^{\mathrm{OP}^{-}}$for $\mathrm{OP}^{-}$and $h_{i}^{\mathrm{SP}^{-}}$for $\mathrm{SP}^{-}$, and we define

$$
x_{i} \triangleq \begin{cases}y_{i}, & \text { for } \mathrm{OP}^{-} \\ y_{i}+2 r_{i}, & \text { for } \mathrm{SP}^{-} .\end{cases}
$$

The only difference from the ordinary SPS method (using optimum curvatures) in [13] is to set $c_{i}$ to zero for $x_{i} \leq 0$ in (18). Nonquadratic penalties are included easily [13].

\section{Simulations}

\section{A. Methods}

To compare the bias and variance properties of the estimators $\left(\mathrm{OP}^{-}, \mathrm{OP}^{+}, \mathrm{SP}^{-}, \mathrm{SP}^{+}\right.$and $\left.\mathrm{SD}\right)$, we simulated a $2 \mathrm{D}$ PET emission scans. The PR model was also included for a comparison purpose since in this simulation we had access to $Y_{i}^{\text {prompt }}$ and $Y_{i}^{\text {delay }}$ separately.

The synthetic emission phantom shown in Fig. 1 was used; its warm background, left cold disc, and right hot disc had relative emission activities of 2, 0.5, and 4, respectively. The sinograms had 192 radial bins and 120 angles uniformly sampled over 180 degrees. The system matrix was generated using ASPIRE [18]; the system geometry was approximated with 3 $\mathrm{mm}$ wide strip integrals and $3 \mathrm{~mm}$ ray spacing. We simulated nonuniform detector efficiencies using pseudo-random lognormal variates with standard deviation of 0.3 . Attenuation was not considered in this simulation. The reconstructed images were 64 by 32 with $9 \mathrm{~mm}$ pixels. The known $r_{i}$ and $s_{i}$ factors corresponded to a uniform field of $60 \%$ randoms and $10 \%$ scatters, respectively.

The specific aim of the simulation was to compare bias for low counts and variance for high counts. We performed two studies with $2 \mathrm{~K}$ and $2 \mathrm{M}$ total counts. We generated 500 realizations of pseudo-random emission measurements according to (1) with (2) and (3). For each realization, images were reconstructed using 100 iterations of the SPS method for $2 \mathrm{~K}$ counts, and using 40 iterations of the SPS method after 10 iterations of ordered subsets SPS (with 8 subsets) [13] for $2 \mathrm{M}$ counts. The FBP reconstruction for each realization served as an initial image for the iterations. For initial FBP reconstructions, a Hanning filter was used with such a cut-off frequency that their local impulse responses were of 3 pixels full-width half-maximum (FWHM).

For regularization, we used a second-order quadratic penalty. It is important to match the spatial resolution in reconstructed images for a fair comparison of different estimators. We used a hybrid technique [19] consisting of two steps: 1) for each method, we adjusted a global regularization parameter $\beta$ so that a local impulse response at the center pixel was of 1.5 pixels FWHM, and then performed PL reconstructions; 2) we applied a 2D Gaussian post-smoothing filter to the 
PL reconstructions so that the overall local impulse response (at the center pixel), which is the convolution of the postsmoothing filter and the original local impulse response (of 1.5 pixels FWHM), achieved a target resolution of 3 pixels FWHM.

This technique enables us to obtain reconstructions with various target resolutions by simply changing the post-smoothing filter. As the post-smoothing filter becomes wider (higher FWHM), the overall resolution becomes more uniform spatially since post-smoothing dominates the overall response. To check the spatial uniformity, the overall resolutions at pixels undersampled by a factor of 3 were investigated and it was found that, except the 2 pixel wide strip along the phantom boundary, each pixel achieved the target resolution (3 pixels FWHM) within 5\% errors for all estimatorsreasonably uniform resolution. The local impulse responses of each estimator were computed using 2D FFT by assuming local shift-invariance [20].

\section{B. Results}

Fig. 1 shows the profiles through the sample mean images of different estimators for $2 \mathrm{~K}$ counts-very low counts. Both $\mathrm{OP}^{+}$and $\mathrm{SP}^{+}$showed positive systematic bias whereas other methods $\left(\mathrm{OP}^{-}, \mathrm{SP}^{-}, \mathrm{SD}\right.$, and $\left.\mathrm{PR}\right)$ seemed reasonably free of such a bias. However, some positive bias in the cold spot (pixels 12-25) is noticeable even for $\mathrm{OP}^{-}, \mathrm{SP}^{-}, \mathrm{SD}$ and $\mathrm{PR}$. This bias is due to the image-domain nonnegativity constraint rather than to zero-thresholding in sinogram (see the large variances of the estimators in Fig. 2). For $\mathrm{OP}^{+}$and $\mathrm{SP}^{+}$, the positive bias in the cold region can be attributed to both zerothresholding and image-domain nonnegativity constraint. Also, note the distinguishably large positive bias of $\mathrm{OP}^{+}$and $\mathrm{SP}^{+}$ near the phantom boundary; the reason for this is that the rays passing the boundary region tend to have lower counts [17]. Overall the systematic bias of $\mathrm{OP}^{+}$was slightly larger than that of $\mathrm{SP}^{+}$.

In Fig. 1, it is also noticeable that FBP shows huge positive bias. This is caused by the large variance of FBP with the image-domain nonnegativity constraint (see Fig. 2).

Fig. 3 shows the profiles through the sample mean images of different estimators for $2 \mathrm{M}$ counts-high counts. All of the methods are seen to be free of systematic biases. Fig. 4 shows profiles through the sample standard deviation images. FBP again showed the highest standard deviation and PR showed the lowest as expected. $\mathrm{SP}^{-}$and $\mathrm{SD}$ showed similar performance, and $\mathrm{OP}^{-}$led to higher standard deviation than both of them. To make a further comparison, we plotted histograms of the ratio of the standard deviation of different methods to the standard deviation of PR in Fig. 5. It also supports the claim that both $\mathrm{SP}^{-}$and $\mathrm{SD}$ lead to less variance than $\mathrm{OP}^{-}$(and FBP).

See [17] for analysis, corroborated by these experimental results, of positive systematic bias (caused by zero-thresholding) and asymptotic variance.

\section{CONCLUSIONS}

We proposed new log-likelihood approximations $\left(\mathrm{SP}^{-}\right.$and $\mathrm{OP}^{-}$) for randoms-precorrected PET emission image reconstruction and also developed algorithms (SPS) for the new models. The new methods are free of positive systematic biases that degrades $\mathrm{SP}^{+}$and $\mathrm{OP}^{+}$images. Our new models seem particularly promising for fully 3D PET emission scans where $\mathrm{AC}$ rates are high and photon counts per ray can be low, essentially for newer scanners with small crystals.

The new $\mathrm{SP}^{-}$model yields less variance (than $\mathrm{OP}^{-}$and FBP). Its performance is comparable to SD as to systematic bias and variance; yet its implementation is simpler.

We recommend the PR method if the prompt and the randoms data are accessible separately; however, if only randomsprecorrected data are available, the new $\mathrm{SP}^{-}$method is our recommended method.

\section{REFERENCES}

[1] E. J. Hoffman, S. C. Huang, M. E. Phelps, and D. E. Kuhl, "Quantitation in positron emission computed tomography: 4 Effect of accidental coincidences," J. Comput. Assist. Tomogr., vol. 5, no. 3, pp. 391-400, 1981.

[2] M. Yavuz and J. A. Fessler, "Statistical image reconstruction methods for randoms-precorrected PET scans," Med. Imag. Anal., vol. 2, no. 4, pp. 369-378, Dec. 1998.

[3] M. Yavuz and J. A. Fessler, "New statistical models for randomsprecorrected PET scans," in Information Processing in Medical Imag., J. Duncan and G. Gindi, Eds., vol. 1230 of Lecture Notes in Computer Science. Berlin: Springer-Verlag, 1997, pp. 190-203.

[4] E. Ü. Mumcuoğlu, R. M. Leahy, and S. R. Cherry, "Bayesian reconstruction of PET images: methodology and performance analysis," Phys. Med. Biol., vol. 41, no. 9, pp. 1777-1807, Sept. 1996.

[5] M. E. Casey and E. J. Hoffman, "Quantitation in positron emission computed tomography: 7 a technique to reduce noise in accidental coincidence measurements and coincidence efficiency calibration," $J$. Comput. Assist. Tomogr., vol. 10, no. 5, pp. 845-850, 1986.

[6] E. Ü. Mumcuoğlu, R. Leahy, S. R. Cherry, and Z. Zhou, "Fast gradientbased methods for Bayesian reconstruction of transmission and emission PET images," IEEE Trans. Med. Imag., vol. 13, no. 3, pp. 687-701, Dec. 1994.

[7] D. Hogg, K. Thielemans, S. Mustafovic, and T. J. Spinks, "A study of bias for various iterative reconstruction methods in PET," in Proc. IEEE Nuclear Science Symp. Medical Imaging Conf., 2002.

[8] D. G. Politte and D. L. Snyder, "Corrections for accidental coincidences and attenuation in maximum-likelihood image reconstruction for positron-emission tomography," IEEE Trans. Med. Imag., vol. 10, no. 1, pp. 82-89, Mar. 1991

[9] M. Yavuz and J. A. Fessler, "Penalized-likelihood estimators and noise analysis for randoms-precorrected PET transmission scans," IEEE Trans. Med. Imag., vol. 18, no. 8, pp. 665-674, Aug. 1999.

[10] M. Yavuz and J. A. Fessler, "Maximum likelihood emission image reconstruction for randoms-precorrected PET scans," in Proc. IEEE Nuclear Science Symp. Medical Imaging Conf., vol. 2, 2000, pp. 15/229$15 / 233$.

[11] S. O. Rice, "Uniform asymptotic expansions for saddle point integralsApplication to a probability distribution occurring in noise theory," Bell Syst. Tech. J., vol. 47, pp. 1971-2013, Nov. 1968.

[12] D. L. Snyder, C. W. Helstrom, A. D. Lanterman, M. Faisal, and R. L. White, "Compensation for readout noise in CCD images," J. Opt. Soc. Amer. A, vol. 12, no. 2, pp. 272-283, Feb. 1995.

[13] H. Erdoğan and J. A. Fessler, "Ordered subsets algorithms for transmission tomography," Phys. Med. Biol., vol. 44, no. 11, pp. 2835-2851, Nov. 1999.

[14] L. A. Shepp and Y. Vardi, "Maximum likelihood reconstruction for emission tomography," IEEE Trans. Med. Imag., vol. 1, no. 2, pp. 113 122, Oct. 1982. 
horizontal profile 17 through sample mean for $2 \mathrm{~K}$ counts

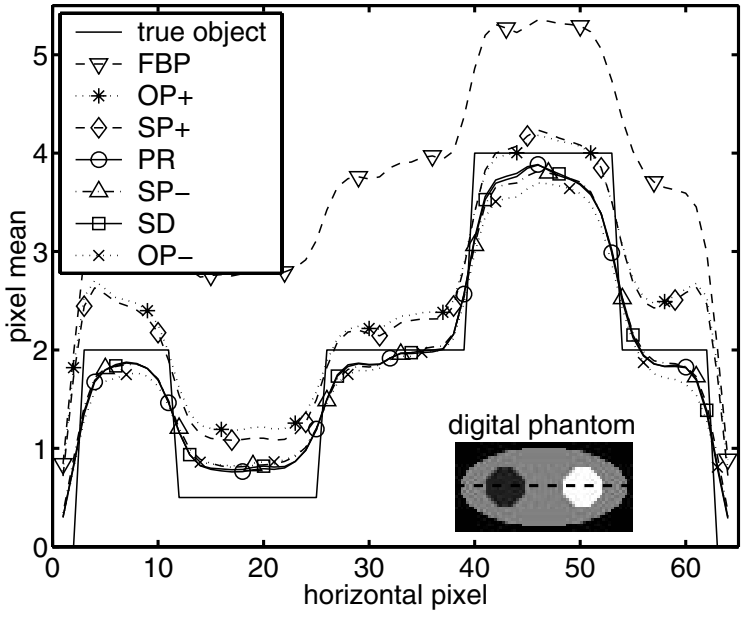

Fig. 1. Horizontal profile through sample mean of estimators for $2 \mathrm{~K}$ counts. The digital phantom used in simulations is shown.

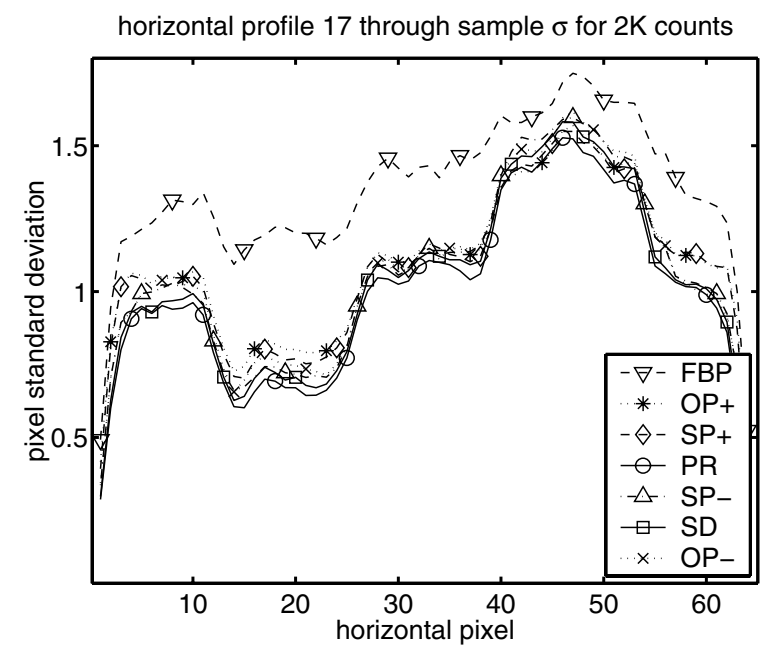

Fig. 2. Horizontal profile through sample standard deviation of estimators for $2 \mathrm{~K}$ counts.

[15] M. Yavuz, "Statistical tomographic image reconstruction methods for randoms-precorrected PET measurements," Ph.D. dissertation, Univ. of Michigan, Ann Arbor, MI, 1999. [Online] Available: http://www.eecs.umich.edu/ fessler/student.html

[16] H. Erdoğan and J. A. Fessler, "Monotonic algorithms for transmission tomography," IEEE Trans. Med. Imag., vol. 18, no. 9, pp. 801-814, Sept. 1999.

[17] S. Ahn and J. A. Fessler, "Emission image reconstruction for randoms-precorrected PET allowing negative sinogram values," IEEE Trans. Med. Imag., submitted for publication. [Online] Available: http://www.eecs.umich.edu/ fessler

[18] J. A. Fessler. (1995, July) ASPIRE 3.0 user's guide: A sparse iterative reconstruction library. Commun. and Signal Proc. Lab., Dept. of Elec. Eng. Comput. Sci., Univ. Michigan, Ann Arbor, Tech. Rep. 293. [Online] Available: http://www.eecs.umich.edu/ fessler

[19] J. W. Stayman and J. A. Fessler, "Compensation for nonuniform resolution using penalized-likelihood reconstruction in space-variant imaging systems," IEEE Trans. Med. Imag., to appear.

[20] J. W. Stayman and J. A. Fessler, "Regularization for uniform spatial resolution properties in penalized-likelihood image reconstruction," IEEE Trans. Med. Imag., vol. 19, no. 6, pp. 601-615, June 2000.

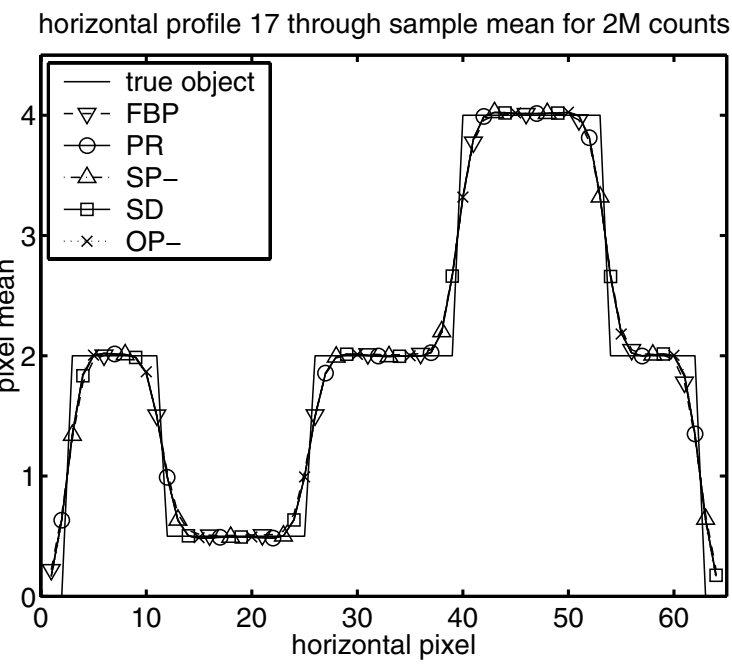

Fig. 3. Horizontal profile through sample mean of estimators for $2 \mathrm{M}$ counts.

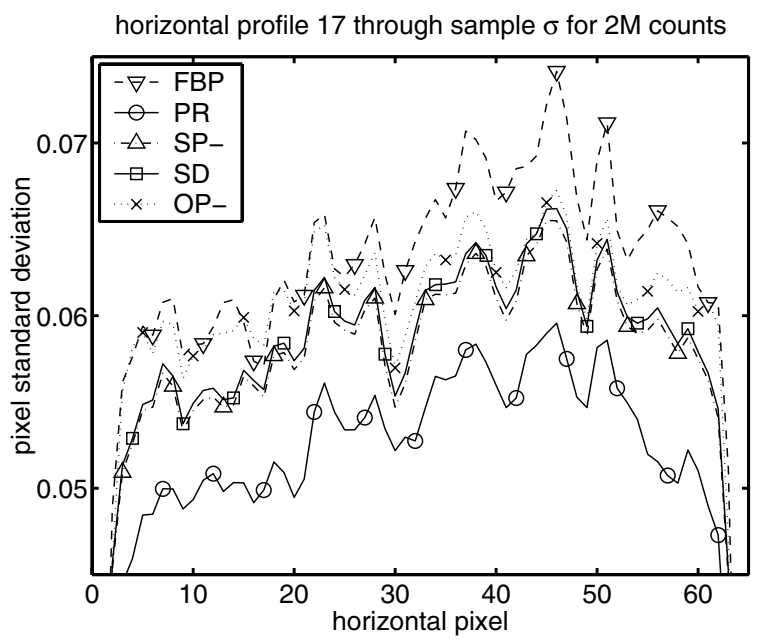

Fig. 4. Horizontal profile through sample standard deviation of estimators for $2 \mathrm{M}$ counts.
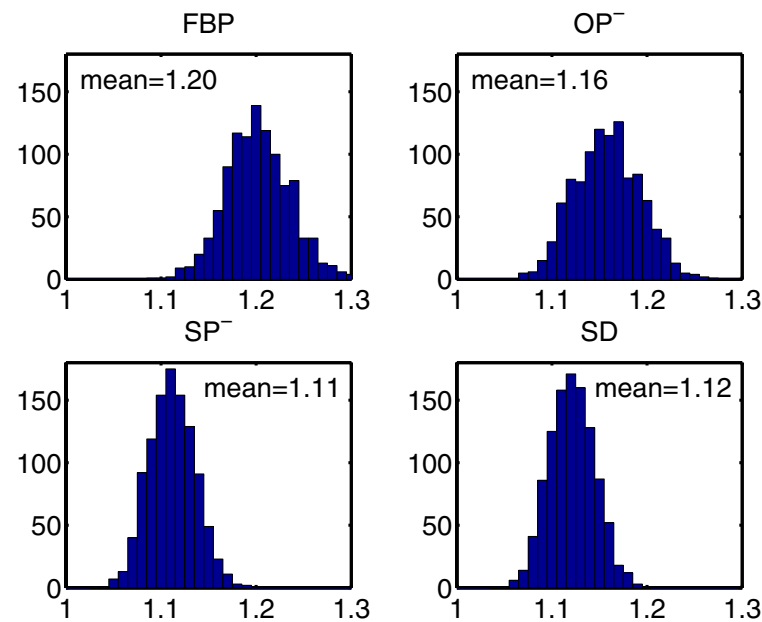

Fig. 5. Histogram of the ratio of the standard deviation of different reconstruction methods to the standard deviation of PR method for $2 \mathrm{M}$ counts 